Animal

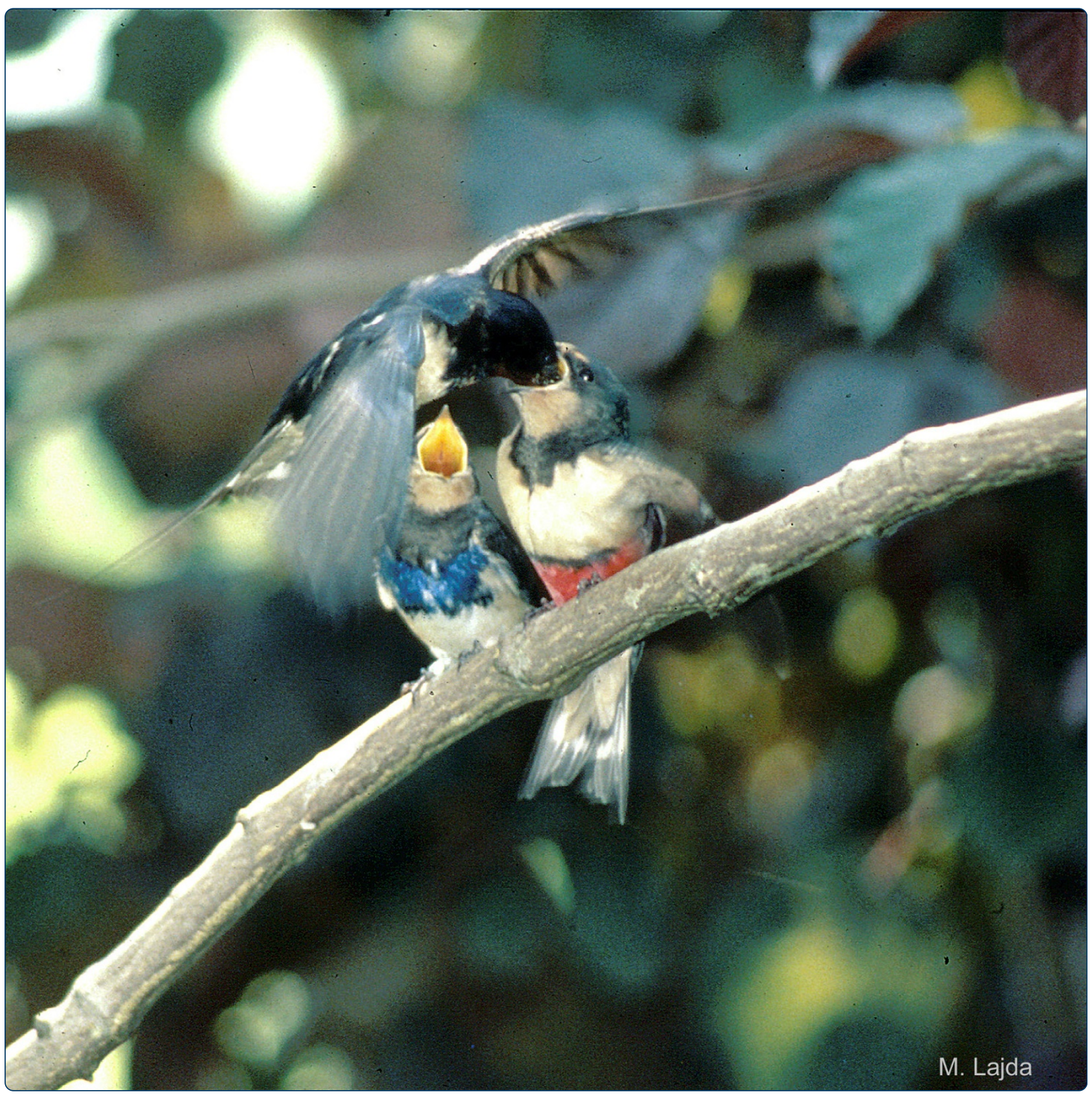

\title{
Effects of radio-tag characteristics and sample size on estimates of apparent survival
}

Naef-Daenzer and Grüebler 


\title{
Effects of radio-tag characteristics and sample size on estimates of apparent survival
}

\author{
Beat Naef-Daenzer ${ }^{*}$ and Martin $\cup$ Grüebler ${ }^{\dagger}$
}

\begin{abstract}
Background: Radio-tracking is increasingly used to assess key characteristics of population dynamics. Since in many species re-encounter rates are frequently below 1.0 and vary with time and/or life-history stages, known-fate approaches to analyses may not apply. Cormack-Jolly-Seber (CJS) models estimate apparent survival on the basis of individual encounter histories. These models allow for complex re-encounter models and constitute an ideal tool to estimate apparent survival where re-encounter rates vary. However, the implications of radio-tag characteristics and sample size on the precision of survival estimates and the potential to determine temporal variation in survival have rarely been investigated. Here we analyze radio-tracking data from juvenile barn swallows (Hirundo rustica) that were instrumented with four types of transmitters similar in mass but differing in radiated power ( $n=560,132$ broods).

Results: For all transmitter types re-encounter probabilities varied between 0.2 and 0.75 , depending on radiated power and bird age. Higher-power transmitters significantly improved the re-encounter rates and thus, the precision of survival estimates. Apparent survival varied with age, with a minimum around the break-up of families. Later on survival increased again and approached the rate of adults. Analyses of random sub-samples revealed that sample size strongly affected the variance in survival estimates, and thus the power in discerning temporal or between-group variation in survival. Small samples substantially underestimated the survival to independence. In small subsamples the standard errors of estimates were particularly large in later re-encounters. Consequently, model selection results of different survival models on the basis of small random sub-samples were highly inconsistent.

Conclusions: Investigating population processes requires modeling of time- and cohort-dependent survival rates, often for short time periods. We show that CJS estimates of apparent survival from small samples revealed rough patterns in barn swallow survival with samples of c. 50 individuals. However, small samples underestimated the number of survivors reaching independence. Inference on underlying mechanistic models based on Akaike's Information Criterion (AIC) model selection was unreliable with sample sizes below 200 individuals. As samples are often limited for practical reasons, maximizing re-encounter rates by optimal choice of telemetry hardware and field logistics is a way to increase the precision of survival estimates.
\end{abstract}

Keywords: Population dynamics, Survival, Cormack-Jolly-Seber, Modeling, Radio-telemetry, Hirundo rustica

\section{Background}

Quantifying survival rates in different populations or in relation to ecological factors and life-history traits is essential for understanding demographic processes. Yet, to assess survival in the field is difficult due to technical problems in observing the fate of individuals. Advanced techniques of mark-recapture analysis allowing separate estimation of apparent survival and re-encounter probability brought a quantum step in disentangling apparent survival

\footnotetext{
* Correspondence: beat.naef@vogelwarte.ch

${ }^{\dagger}$ Equal contributors

Swiss Ornithological Institute, Seerose 1, CH-6204 Sempach, Switzerland
}

from missed re-observations. Thus, parameter estimates can be corrected for variation in the re-observation probability of individuals among cohorts, in relation to time and to other covariates. The models are built on the basis of individual encounter histories ([1], applicationrelated review in [2]). As very low detection rates still impose a principal problem, technical means to improve the re-encounter rate are crucial where individuals are difficult to detect (for example, on the sea, at night, in dense vegetation, [3]). Very high frequency (VHF) telemetry is increasingly used to assess key parameters of population dynamics, such as differential survival in relation to multiple 
factors, such as sex, age, season and life history traits (for example, dispersal and immigration behavior). In particular, the technique improves the re-encounter rate of animals, makes re-encounters independent of visibility conditions, and is thus often preferred over alternative marking techniques. However, detection probabilities in field studies are often markedly below 1.0 and thus, insufficient for the application of known-fate analyses. Transmitter characteristics, mainly radiated power, life and duty cycle likely affect the efficiency of the technique (for example, $[4,5]$ ). To assess critical periods in the life history of species it is often desired to quantify survival rates for short periods of time (for example, the post-fledging period of birds). To our knowledge, the implications of transmitter technical characteristics for collecting survival data in the field and for survival analysis have not been addressed quantitatively. While it is standard practice to evaluate potential adverse effects of radio-tagging on study animals [4] or to test effects of sample size on home-range estimates [4], two important issues remain unclear: first, the technical characteristics of radio tags, specifically their radiated power, may have a strong impact on the probability of detecting individuals. Within a study, transmitters of different power and life are often used in parallel, and all battery-driven radio-tags lose power towards the end of transmitter life. This is expected to cause changes in the detection probability of individuals irrespective of their behavior or survival, thus violating the assumption that $p$ is close to 1.0 and constant over a study period. Second, the impact of sample size (and detection rate) on estimating apparent survival and its importance for inference upon model selection (using Akaike's Information Criterion (AIC)) remain largely unclear [6,7], but see [8]. So far, evidence-based rules for evaluating the inferential power in discerning between-cohort or temporal variation in survival estimates for different cohorts or time periods are lacking.

Here, we use the barn swallow (Hirundo rustica L.) as an example organism to analyze how variation in the encounter technique and sample size affects the inferential power of Cormack-Jolly-Seber (CJS) model selection. Specifically, we first test whether the re-encounter technique (that is, color marks and radio-tags of different radiated power) affects the re-encounter rates during the early life-history stages of the study organism, particularly during the post-fledging period and after family breakup. Second, we evaluate the effects of sample size on estimates of survival, which is the target variable of biological importance. In the barn swallow, assessing shortterm variation in apparent survival during the post-fledging period is particularly important. Our general approach to the problem is by subsampling the large sample $(n=560)$ of tracked birds. Given the availability of a large sample of real fates, we preferred a re-sampling approach over the simulation of data. General statistical theory predicts that the uncertainty of estimates decreases with sample size (that is, precision increases). To evaluate the shape of this relationship quantitatively is an important issue for planning data collection and field logistics in order to test between-group and temporal variation in apparent survival.

Our analysis emphasizes that both the re-encounter technique and sample size have a pervasive influence on the inferential power of CJS model selection, and thus, on drawing conclusions on demographic processes. It also contributes to resolve important trade-offs in study design; as most field studies face severe financial and logistic restrictions, the results provide means to plan studies to yield the desired results without imposing excessive costs.

\section{Results}

Transmitter effects on re-encounter rates

The top ranked model using the full sample showed a strong effect of both time after fledging and type of transmitter (color-marked, tags differing in radiated power) on re-encounter probabilities. Figure 1 illustrates that even with the use of radio-tags, re-encounter rates were low and that the radiated power of the tags strongly influenced the

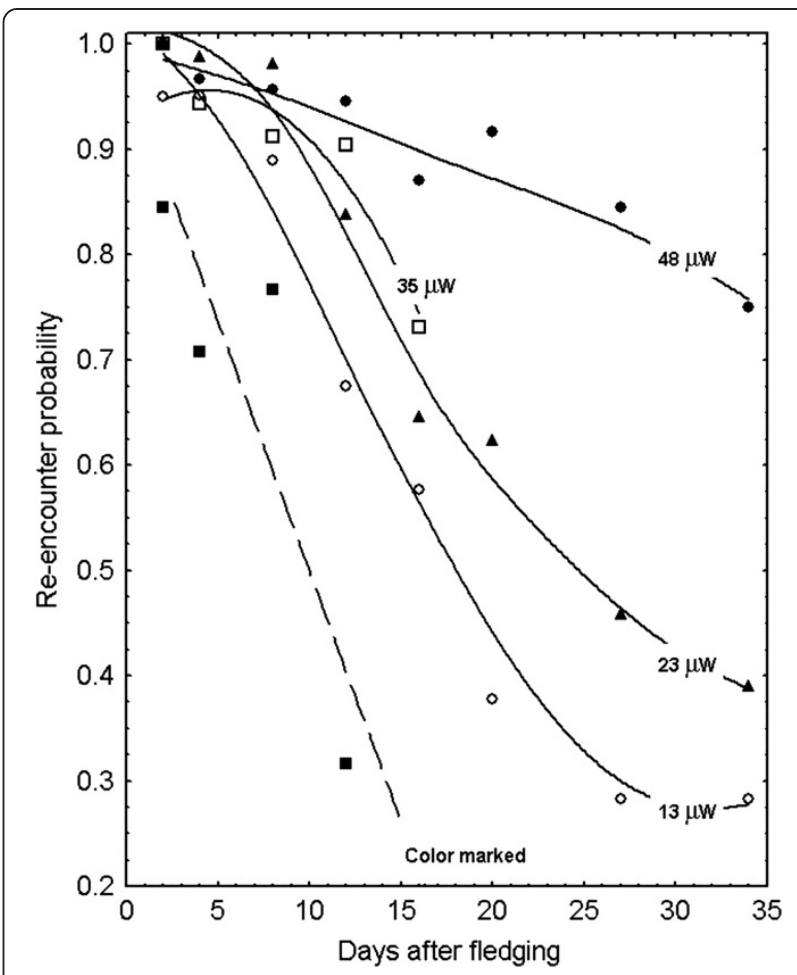

Figure 1 The re-encounter probabilities $(p)$ in relation to time after fledging as calculated using the full sample. Transmitter specifications and cohort sizes are given in Table 1. Color-marked birds were not recovered regularly after family break-up, that is, 12 to 15 days after fledging. The life of a $35 \mu \mathrm{W}$ tag was less than $20 \mathrm{~d}$. Trend lines were fitted by distance-weighted least squares, except for color marks (linear trend). 
probability of re-encountering individuals. Over all age classes the re-encounter rate of color-marked individuals was lowest. In the first days after fledging, the daily reencounter rate for color-marked birds was about 0.8 . However, this rate declined quickly with age and dropped to c. 0.3 before the families broke up. After family breakup, recoveries of color-marked individuals occurred only occasionally ( $p$ approximately 0 ). Compared to color marks, all radio-transmitters improved the re-encounter rates, and made individuals observable reliably beyond family break-up. In the first five days after fledging the re-encounter rate of radio-tagged individuals was close to 1.0 with all types of radio-tags. In this period the range used by families was small but the chicks frequently hid in trees and bushes near the farms. Hence, while the radio signal was crucial for detecting the birds, transmitter power (range) was less. In contrast, from post-fledging Day 6 , the families and, later on, the independent juveniles, moved quickly over distances of up to $15 \mathrm{~km}$. Accordingly, the re-encounter rate decreased in relation to age and increased with transmitter power. The daily re-encounter rate after one month was $0.28 \pm 0.07$ s.e. with $13 \mu \mathrm{W}$ transmitters, $0.39 \pm 0.07$ with $23 \mu \mathrm{W}$ transmitters and $0.75 \pm 0.08$ with transmitters radiating $48 \mu \mathrm{W}$ (Figure 1).

\section{Sample size effects on survival estimates}

The respective top-ranked models for the 50 sub-samples and for the full sample returned roughly similar temporal patterns in the estimates for apparent daily survival (for details on sub-sampling see methods section). As with the full-sample model, sub-samples revealed declining daily survival rates in the first days after fledging (re-encounters 2 to 5) with a minimum around Day 15 post-fledging. Then the rates increased again for fledglings older than 16 days post-fledging (Figure 2). Since all birds surviving to the entire observation period remained in the study area, we exclude that the temporal variation in apparent survival was affected by temporal emigration or similar behavior. However, the slight decline in apparent survival in the last re-encounter interval (post-fledging days 31 to 40) may be due to chicks of late second broods leaving the study area for migration shortly after reaching independence.

The variation in results obtained from small subsamples was large (Figure 2a). On average, small subsamples dramatically underestimated the proportion of birds surviving to the end of the observation period. With sub-samples of less than 50 individuals, the proportion of juveniles surviving to post-fledging Day 40 was estimated at $0.086 \pm 0.043$, whereas large subsamples $(>250)$ indicated a total survival of $0.285 \pm 0.012$ (Figure $2 \mathrm{~b}, \mathrm{~F}_{21,184}=4.8, P<0.0001$ ). We conclude from this that the estimates for each re-encounter interval as obtained from small subsamples were slightly biased to the negative.
As expected from statistical theory, the variation of the estimates of both apparent survival and re-encounter rate increased inversely to sub-sample size (Figures 2 and 3a). These 'trumpet-shaped' relationships indicate that the error of parameter estimates grows very large with samples of less than c. 100 individuals. For small sub-samples we found a marked negative correlation between the estimate for the re-encounter rate and that of apparent survival (Figure 3b). Accordingly, as compared to the full sample, under-estimation of the encounter rate $(P)$ resulted in substantial over-estimation of survival $(\Phi)$. With increasing sample size, both estimates converged to a small range, and thus, the correlation between $P$ and $\Phi$ disappeared (black dots in Figure $3 \mathrm{~b}$ ). However, the estimated survival rates were consistently above the regression line obtained from small sub-samples. This apparent bias was particularly large with samples of less than 100 individuals (light gray dots in Figure 3b. These results indicate that, in addition to some bias, the precision of point estimates strongly decreased with decreasing sample size. Figure 4 illustrates that these effects strongly influenced the quality of survival estimates, the target variable of CJS modeling. In particular, in small subsamples, the uncertainty of estimates for later re-encounters was very high. Only in sub-samples of over 250 individuals the standard errors of survival estimates were homogeneous over the entire observation period (contour lines in Figure 4).

Comparing the favored models for the 10 entirely different sub-samples of 50 individuals each (that is, subsamples drawn without replacement) further emphasized that variation in the encounter histories within small sub-samples strongly affected the outcome of model selection. Out of the eight 'simple' models (that is, those implemented in the software MARK without group encounter interactions, see methods section) five were favored at least once. The respective corrected AIC with quasi-likelihood adjustment (QAICc) weights ranged from 0.549 to 0.921 , and in 8 of the 10 cases the difference in QAICc $(\triangle \mathrm{QAICc})$ to rank 2 exceeded 2.0. With regard to apparent survival, the conclusions drawn upon one particular sub-sample would therefore differ considerably: 1) the results from five sub-sets would suggest that apparent survival was constant over groups and re-encounters $(\Phi()), 2$.$) three sub-samples would$ indicate that apparent survival differs among transmitter types but not among re-encounters $(\Phi(\mathrm{g}))$, and 3) two sub-samples would suggest that apparent survival differs among encounters but not in relation to transmitter type $(\Phi(\mathrm{t}))$. We conclude from this that model selection on the basis of a sample of 50 individuals was highly sensitive to the particular composition of encounter histories, and thus insufficient to represent the statistical population. 

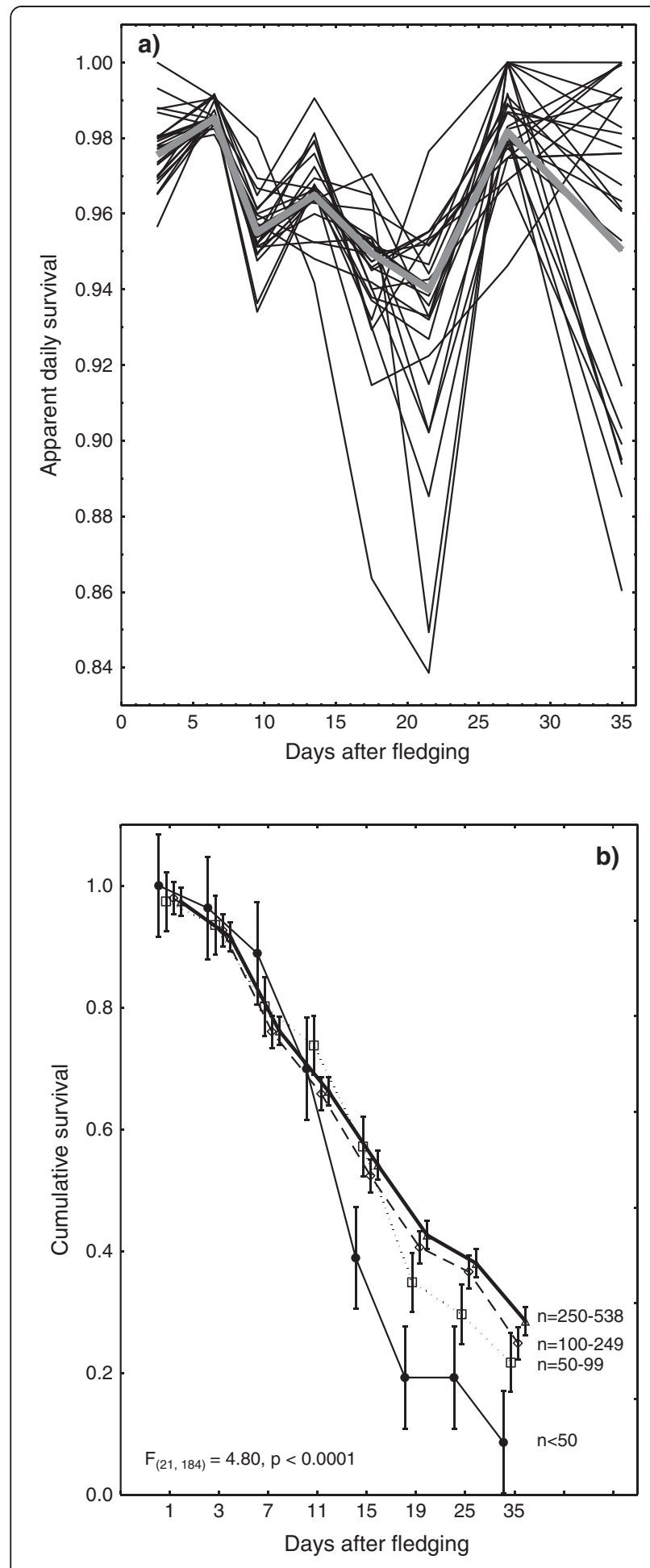

Figure 2 Estimates of daily apparent survival $(\Phi)$ from all sub-sample models for post-fledging days 1 to 40 . a) Daily apparent survival rates (one line per model). Most models reveal a drop in daily survival around 15 days post-fledging; however, the variation in point estimates is large and several samples deviated strongly from the general pattern. The bold gray line indicates estimates from the full sample size $(n=538)$. b) Cumulative post-fledging survival as estimated from four categories of sub-samples size in relation to time postfledging ( $x$-axis gives category means). Small subsamples dramatically underestimate the proportion of birds surviving to the end of the observation period. Dots and whiskers give means and 95\% confidence intervals per sample size category and re-encounter interval.

\section{Discussion}

\section{Parameter estimates}

The analyses and simulations revealed that CJS-models based on small sub-samples of individuals yielded inconsistent estimates of apparent survival compared to the results from the full sample of 538 radio-tagged birds. Models using small sub-samples deviated substantially from the general temporal pattern in survival rates and strongly underestimated the proportion of individuals surviving to the end of the observation period (Figure 2). The main source of these deviations was likely the imprecise estimation of re-encounter rates. In a small sample, the estimates of $p$ depend much on occasionally missing one single animal in a particular encounter interval. Thus, estimates of apparent survival may be seriously biased due to inaccurate modeling of re-encounter rates (Figure 3b, [9]). This leads to the first main conclusion that optimal encounter techniques may be an important measure to maximize the precision of estimates from a given sample size. With sub-samples of less than 50 individuals, the uncertainty of estimates of the proportion of juveniles surviving to Day 40 post-fledging was so large that estimating cumulative survival over the observation period (that is, the number of chicks surviving to independence) was strongly biased (Figures 2 and 4). We therefore suggest that, particularly if increasing sample size is impractical, care is given to maximizing the quality of data. This implies maximizing the encounter probability (for example, by increasing transmitter power) and by increasing the rate of re-encounter attempts or the duration of searches per re-encounter interval. Increasing the re-encounter intervals is another option. However, where the short-term variation in apparent survival in relation to specific life-history stages is in the focus (this study, [10]), extending the re-encounter intervals is often undesirable. Also, collecting additional evidence, such as dead recoveries, supporting survival estimates may improve the inferential power of analyses of small samples.

\section{Model selection}

Model selection was highly sensitive to the particular composition of the 10 small sub-samples drawn without 

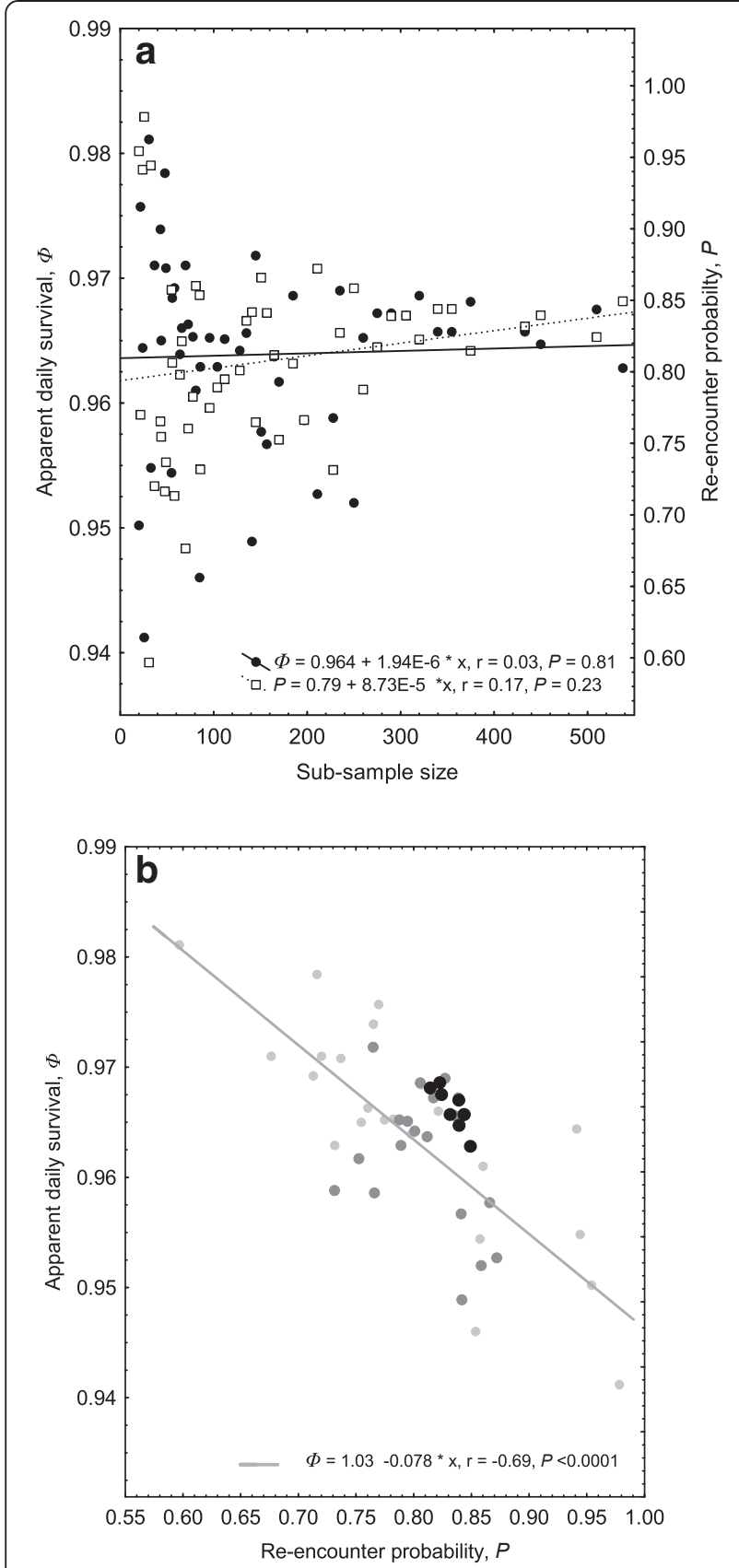

Figure 3 Precision and bias in estimates of apparent survival $(\boldsymbol{\Phi})$ and re-encounter rate $(\boldsymbol{p})$. a) Estimates of $\Phi$ and $p$ in relation to sub-sample size. In both parameters the variation of estimates strongly increased inversely to sub-sample size. b) Average estimates of apparent survival in relation to re-encounter probability. Small sub-samples were likely to give biased estimates of the re-encounter rate and in turn, local survival. Dot size and color indicate sample size categories (small light gray $n \leq 100$, medium, gray $n=101$ to 300 , large, black $n>300$ ). The line gives the regression for samples of $n<100$ individuals. With large sample sizes the estimates converge to a narrow range and the correlation disappears.

replacement. Point estimates for groups and/or encounters obtained from small samples may, therefore, deviate substantially from the statistical population. Thus, the

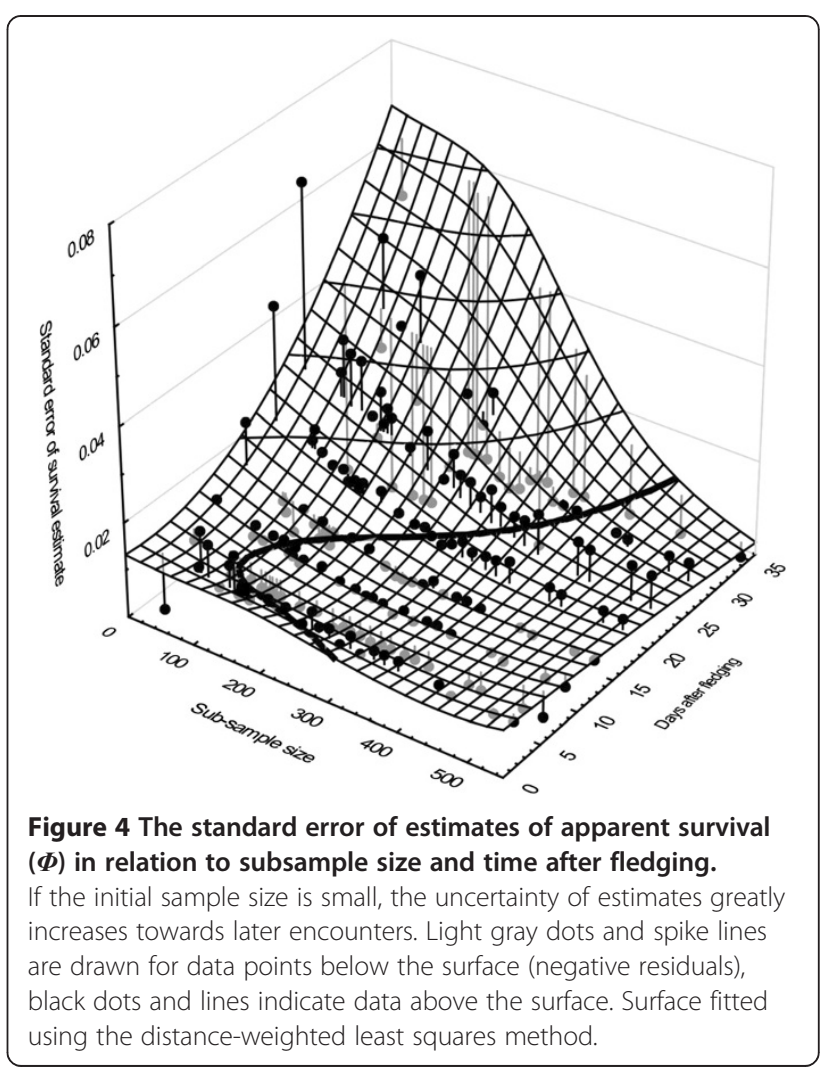

second main conclusion is that, where only small samples of individuals (below c. 200 individuals) can be included, inference upon a specific model and on underlying biological mechanisms, should be drawn very carefully, and requires support by additional evidence $[7,9]$. Even if the difference in QAICc to lower ranked models was large $(>2.0)$, alternative models were difficult to discern due to the uncertainty in estimates for both parameters. One system-immanent cause for these inconsistent results of model selection is that all cohorts gradually decline in numbers due to mortality. Therefore, if the initial sample is small, the basis for estimates at later re-encounters declines to a very few individuals, hence to have missed or encountered one individual at a particular occasion has a strong impact on the modeling results ([11,12] Figure 4). This effect increases with decreasing re-encounter probability and increasing re-encounter intervals.

\section{Conclusions}

Recent advances in radio-telemetry have opened access to studying important problems in population dynamics, such as differential survival during life-history stages in which individuals are difficult to observe (for example, $[10,13,14])$. However, for practical reasons many empirical studies are restricted to relatively small samples of individuals. This investigation indicates that the inferential power 
of AIC model selection based on small samples may be weaker than often presumed for sample sizes of below c. 50 individuals. Particular problems arise in estimating survival parameters for later encounters, when the initial sample gradually declines (Figure 4). Therefore, to obtain optimal survival estimates, specifically to quantify differentials in relation to specific cohorts, to time or particular traits, we suggest that maximizing the sample size as much as affordable is of paramount importance. In parallel, using the best encounter technique and optimal modeling of the re-encounter rate $p$ may greatly improve the accuracy of survival estimates and, thus, the inferential power of analyses. These measures may not only include the selection of optimal telemetry hardware, but also careful planning of field techniques and manpower required for data collection.

\section{Methods}

The data were collected in a study on the post-fledging survival and range use of juvenile barn swallows that was carried out in 2000 (pilot study) and 2002 to 2004 (full project) in the Wauwilermoos area, an intensively cultivated plain of c. $20 \mathrm{~km}^{2}$ near Lucerne, Switzerland ( $47^{\circ} 10^{\prime} \mathrm{N} / 8^{\circ} 02^{\prime} \mathrm{E}$ ). Data of 560 barn swallow fledglings of 132 first and second broods at 60 farms were included. Further details on the study area and the study population are given in [15].

\section{Radio-tagging}

We caught barn swallow fledglings at their nest during the night of nestling Day 19 or 20. In 2000 (pilot study) the chicks were radio-tagged with Holohil LB2 transmitters (0.7 g, Holohil Inc., Carp, ON, Canada). In 2002 to 2004 we used radio-tags of our own construction [16,17]. These differed from the LB2 transmitters in an extended life of $30 \mathrm{~d}$ (2002) to $55 \mathrm{~d}$ (2004). Due to technical improvements, the radiated power of these tags increased considerably from 2002 to 2004 (Table 1, [17]). All radio-tags (including battery and harness) had a mass of 650 to $750 \mathrm{mg}$, which represents 3.8 to $4.4 \%$ of the minimum fledgling mass (17 g).

The transmitters $(n=538)$ were attached using a Rappoletype harness made from $0.5 \mathrm{~mm}$ elastic cord $[18,19]$. The juveniles were also individually color-marked on the light plumage of the belly to allow identification after potential loss or failure of transmitters. A combination of two colors out of four (green, blue, red, none) was applied using waterproof pencil (Edding 3000 ${ }^{\circ}$ Edding Inc., Ahrensberg, Germany). These patterns occurred repeatedly among families. Additionally, a family-specific mark was applied. To prevent premature departure the nest cups were closed using a flexible sheet of black plastic. The marked chicks were set back into the nest in complete darkness. We then waited 5 to 10 minutes until the birds had relaxed, and removed the cover.

It is important to ascertain that the marking technique has no effect on the target parameter of a study, which in this study was survival. Earlier studies did not find adverse effects of radio-tagging on small birds of c. $20 \mathrm{~g}$ (adult barn swallow [20]; great and coal tit [21]). Based on this evidence, we included only a small control group of untagged but color-marked birds. The treatment of these birds $(n=22)$ was identical to the above procedure except that no transmitter was attached. The most parsimonious model of swallow survival rates on the basis of the full sample ( $\mathrm{n}=538$ radio-tagged birds) included a relationship between survival rate and time after fledging. Adding an effect of the type of marking (radiotag vs. color mark) on survival did not improve parsimony (Table 2). Including an effect of tagging on survival resulted in a slight increase of the QAICc. However, in both models discerning color-marked and radio-tagged birds (ranks 2 and 3, Table 2) the differences in survival estimates for color-marked and radio-tagged individuals were very small (average daily survival over the first 15 days from fledging $0.956 \pm 0.006$ (s.e.) in radiotagged and $0.932 \pm 0.022$ (s.e.) in color-marked birds, respectively). This further corroborates that radio-tagging had no measurable adverse effect in terms of apparent survival.

Table 1 Sample sizes and technical specifications for the different types of markings used in the study

\begin{tabular}{|c|c|c|c|c|c|}
\hline Year & Mark type & Radiated power & Tag life, $d$ & $\mathrm{~N}$ & Comment \\
\hline 2000 & Color mark & & & 14 & \\
\hline 2002 & & & & 8 & \\
\hline 2000 & Holohil LB2 & $35 \mu \mathrm{W}(-15.2 \mathrm{dBm})$ & 13 to 21 & 46 & www.holohil.com \\
\hline 2002 & Own, one stage & $13 \mu \mathrm{W}(-18.5 \mathrm{dBm})$ & 30 to 35 & 203 & ZA10 ZnO2 cells \\
\hline \multirow[t]{2}{*}{2003} & Own, one stage & $23 \mu \mathrm{W}(-16.3 \mathrm{dBm})$ & 30 to 35 & 203 & 396 AgO cells \\
\hline & & & & & Harness on transmitter \\
\hline \multirow[t]{2}{*}{2004} & Own, one stage matched antenna & $48 \mu \mathrm{W}(-13.1 \mathrm{dBm})$ & 50 to 60 & 86 & $396 \mathrm{AgO}$ cells \\
\hline & & & & & Harness on cell \\
\hline
\end{tabular}


Table 2 Model selection for post-fledging survival probabilities of barn swallows

\begin{tabular}{|c|c|c|c|c|c|}
\hline Model & QAICc & $\triangle \mathrm{QAICc}$ & QAICc weight & $\mathrm{np}$ & Deviance \\
\hline$\Phi_{(\text {age })} p_{\text {(type.age) }}$ & 5904.93 & 0.00 & 0.481 & 47 & 5810.22 \\
\hline$\Phi_{(\text {tag }+ \text { age })} P_{\text {(type-age })}$ & 5905.34 & 0.42 & 0.391 & 48 & 5808.61 \\
\hline$\Phi_{\text {(tag•age) }} P_{\text {(type.age })}$ & 5907.57 & 2.65 & 0.128 & 52 & 5802.71 \\
\hline$\Phi_{(\text {age })} p_{(\text {type+(tag.age }))}$ & 5930.86 & 25.94 & 0.000 & 26 & 5878.64 \\
\hline$\Phi_{(\text {age })} P_{(\text {type }+ \text { age })}$ & 5935.14 & 30.21 & 0.000 & 20 & 5895.01 \\
\hline$\Phi_{\text {(age) }} p_{\text {(tag.age) }}$ & 6094.60 & 189.67 & 0.000 & 23 & 6048.43 \\
\hline$\Phi_{(\text {age })} p_{(\text {tag }+ \text { age })}$ & 6094.69 & 189.77 & 0.000 & 16 & 6062.61 \\
\hline$\Phi_{(\text {age })} p_{(\text {age })}$ & 6180.66 & 275.73 & 0.000 & 15 & 6150.58 \\
\hline$\Phi_{(\text {age })} p_{(.)}$ & 6700.32 & 795.39 & 0.000 & 9 & 6682.29 \\
\hline
\end{tabular}

For each model, the value of deviance, number of parameter (np), the corrected Akaike's Information Criterion with quasi-likelihood adjustment (QAICc) and the QAICc-deviation to the best model ( $\triangle \mathrm{QAICC}$ ) are given. For all models an overdispersion factor $\hat{c}=1.192$ was used. Model notation: $\Phi$, survival probability; $p$ re-encounter probability; +, additive effect; $\cdot$, interaction; tag, radio-tagged/color marked; type, four categories of radiated power.

\section{Encounter histories}

The families and independent juveniles were located twice per day. The observation sessions lasted one hour and included the location and visual identification of the birds, and the collection of behavioral data. Missing birds were searched within an area of approximately $100 \mathrm{~km}^{2}$ using fixed antenna stations on vantage points and by using vehicles equipped with an omni-directional antenna. Additionally, flocks of swallows in the study area and groups of birds roosting inside buildings were searched for individuals with color-marks. We never recorded radio-tagged birds leaving the area within the first three weeks post-fledging. Even after wider excursions, all birds returned close (1 to $2 \mathrm{~km}$ ) to the nest sites. During weeks 4 to 5 post-fledging, the birds regularly roosted in nearby wetlands. Thus, juvenile barn swallows did not emigrate from the area up to this age, except a few individuals from late second broods that may have left the study area for migration (Figure 2, latest encounter interval). All telemetry and visual observations were used to build daily encounter histories for the analysis of survival and re-encounter rates (fledging day $0=$ first encounter).

\section{Statistical analyses}

The central issue is that even with the use of radio-tags, re-encounter rates for individuals are normally below 1.0 and may vary largely, since signals may be temporarily missed although the individual is present and alive. For example, topographic characteristics of the study area can restrict the detection ranges, individuals may hide in places that shield radiation (for example, underground or in tree holes), and adverse weather conditions (for example, heavy rain) may temporarily reduce the detection probability of transmitters. Also, animals may move temporally out of the operational range of the transmitter but not disperse from the study area. Setting of transmitter duty cycles too restrictively may also result in missed re-encounters [5].

We used Cormack-Jolly-Seber (CJS) mark-recapture models $[1,22]$ in the software package MARK [23]. The individual encounter histories were used to model probabilities of apparent survival $(\Phi)$ and re-encounters $(p)$. We aggregated encounter histories for 0 time intervals of 1 to 10 days each (post-fledging days 1, 2 to 5,6 to 9, 10 to 13,14 to 17,18 to 21,22 to 28,29 to 40 ; Day 0 = fledging day, figures indicate the midpoints of each re-encounter interval). Encounter histories were grouped according to the type of marking (type: color-marked, $13 \mu \mathrm{W}, 23 \mu \mathrm{W}, 35 \mu \mathrm{W}, 48 \mu \mathrm{W})$. A goodness-of-fit test was performed on the basis of a highly parameterized model, including variation in survival in relation to age and differences between first and second broods, and variation of the re-encounter rate in relation to age and transmitter type, but excluding interactions ( $\Phi_{\text {(brood.age) }}$ $\left.p_{\text {(type age) }}\right)$. We performed a parametric bootstrap with $\mathrm{n}=1,000$ replicates. The observed deviance (dev. $=3,025.14)$ did not differ significantly from the simulated deviances (mean dev. $=2,950.38 \pm 10.04$ s.e., $P=0.22$ ), indicating that our set of models adequately fitted the data. The over-dispersion factor $\hat{c}$ was 1.192. Slight over-dispersion was likely to occur, since cohorts were not equal in size, and fledglings of the same family were treated as independent (see also [22]). In contrast to data obtained from capture-mark-recapture by fixed trap sites, data obtained from radio-tracking are not area-sensitive because the search area can be adjusted to the animals' behavior; estimates of apparent survival are not affected by variation in individual range use. Thus, while re-encounter rates of wide-ranging individuals may decrease, estimates of apparent survival are not biased by altered range use. Estimates of apparent survival are thus relative to the maximum area under survey.

\section{Sub-sampling}

To simulate the effects of sample size on the estimates of survival $(\Phi)$ and re-encounter rate $(p)$ we drew a total of 50 sub-samples of varying size from the full sample of 538 radio-tagged individuals. Random sub-samples were drawn with replacement and the individual histories were randomly re-arranged using the random number generator in the software Excel (Microsoft Switzerland GmbH, Bern, Switzerland). To further evaluate the performance of model selection we split the full sample into 10 random sub-samples of 50 individuals each, that is, without replacement. For all sub-samples, the 16 pre-defined models implemented in MARK were compared (combinations of the following encounter and survival models: constant: $\Phi(.) / p($.$) ; time(age)-dependence: \Phi(\mathrm{t}) / p(\mathrm{t})$; tag-dependence: $\Phi(\mathrm{g}) / p(\mathrm{~g})$; time- and tag-dependence 
(interaction): $\left.\Phi\left(\mathrm{g}^{*} \mathrm{t}\right) / p\left(\mathrm{~g}^{*} \mathrm{t}\right)\right)$. The over-dispersion factor $\hat{c}$ of 1.192 was also applied to these models.

Capture, radio-tagging and tracking was conducted under license of the Swiss Federal Offices for the Environment and for Communication (1000130413.05, technical license; F044-0799 ringers' license).

\section{Abbreviations}

AIC: Akaike's information criterion; QAICc: correctedAIC with quasi-likelihood adjustment; $\triangle$ QAICc: difference in QAICc between ranked models;

CJS: Cormack-Jolly-Seber.

\section{Competing interests}

Neither BND nor MG have any competing interests.

\section{Authors' contributions}

BND and MG conducted the study in close cooperation at all levels. Both contributed equally to planning, data collection, analysis and writing. Both authors read and approved the final manuscript.

\section{Authors' information}

BND is head of and MG is senior researcher in the Ecology Research Group at the Swiss Ornithological Institute.

\section{Acknowledgements}

Many assistants contributed to the study by collecting data on the survival and behavior of birds: S. Aschwanden, P. Contesse, S. Ehrenbold, K. Gatzsch, B. Gerber, E. Glaus, W. Göggel, A. Hüsler, J. Huber, S. Knecht, M. Knöpfel, G. Kraus, P. Kunz, S. Küttel, M. Lajda, J. Morales, L. Naef-Daenzer, D. Nold, M. Nuber, F. Peretti, B. Reichhart, M. Ritschard, S. Schröter, D. Sidler, N. Signorell, U. Suter, L. von Dach, R. Wagner and P. Wetli. Sixty farm families permitted access to their buildings, even at night. L. Jenni and M. Kéry provided comments on the manuscript which was further improved by the comments of two reviewers and B. Collier. This research was funded by the Swiss National Science Foundation (Grant 3100-65382.01 to B. Naef-Daenzer).

Received: 28 May 2013 Accepted: 28 November 2013

Published: 27 January 2014

\section{References}

1. Lebreton JD, Burnham KP, Clobert J, Anderson DR: Modeling survival and testing biological hypotheses using marked animals: a unified approach with case studies. Ecol Monogr 1992, 62:67-118.

2. Nichols JD, Kendall WL, Runge MC: Estimating survival and movement. In Bird Ecology and Conservation. Edited by Sutherland WJ, Newton I, Green RE. Oxford, UK: Oxford University Press; 2004:119-139.

3. Murray DL: On improving telemetry-based survival estimation. J Wildl Manage 2006, 70:1530-1543.

4. Kenward RE: A Manual for Wildlife Radio Tagging. London: Academic Press; 2001.

5. Nicholls DG, Robertson CJR, Naef-Daenzer B: Evaluating distribution modeling using kernel functions for northern royal albatrosses (Diomedea sanfordi) at sea off South America. Notornis 2005, 52:223-235.

6. Mayer MS, Fuller TK, Deblinger RD, McDonald JE: Can low-precision population and survival estimates be accurate? Wildl Soc Bull 2002, 30:440-448.

7. Lindberg MS, Walker J: Satellite telemetry in avian research and management: sample size considerations. J Wild Manage 2007, 71:1002-1009.

8. Skalski JR, Seaburg AG, Buchanan RA: The effects of high detection probabilities on model selection in paired release-recapture studies in the era of electronic tagging studies. Anim Biotelem 2013, 1:12.

9. Zabel RW, Wagner T, Congleton JL, Smith S, Williams JG: Survival and selection of migrating salmon from capture-recapture models with individual traits. Ecol App 2005, 15:1427-1439.

10. Grüebler MU, Naef-Daenzer B: Fitness consequences of pre- and post-fledging timing decisions in a double-brooded passerine. Ecology 2008, 89:2736-2745.

11. Burnham KP, Anderson DR: Data-based selection of an appropriate biological model: the key to modern data analysis. In Wildlife 2001 Populations. Edited by McCullagh DR, Barrett RH. London: Elsevier; 1992.

12. Rexstad EA, Burnham KP, Anderson DR: Design of survival experiments using marked animals: a case study. Am Fisheries Soc Symp 1990, 7:581-587.
13. Moehrenschlager A, Macdonald DW: Movement and survival parameters of translocated and resident swift foxes Vulpes velox. Anim Conserv 2003, 6:199-206.

14. Melnychuk M: Estimation of survival and detection probabilities for multiple tagged salmon with nested migration routes using a large-scale telemetry array. Mar Freshwater Res 2010, 60:1231-1243.

15. Grüebler MU, Naef-Daenzer B: Postfledging parental effort in barn swallows: evidence for a trade-off in the allocation of time between broods. Anim Behav 2008, 75:1877-1884.

16. Naef-Daenzer B: A new transmitter for small animals and enhanced methods of home-range analysis. J Wildl Manage 1993, 57:68-689.

17. Naef-Daenzer B, Früh D, Stalder M, Wetli P, Weise E: Miniaturization (0.2 grams) and evaluation of attachment techniques of telemetry transmitters. J Exp Bio/ 2005, 208:4063-4068.

18. Rappole JH, Tipton A: New harness design for attachment of radio transmitters to small passerines. J Field Ornithol 1990, 62:335-337.

19. Naef-Daenzer B: An allometric function to fit leg-loop harnesses to terrestrial birds. J Avian Biol 2007, 38:404-407.

20. Brigham RM: Effects of radio transmitters on the foraging behavior of barn swallows. Wilson Bull 1989, 101:505-506.

21. Naef-Daenzer B, Widmer F, Nuber M: A test for effects of radio-tagging on survival and movements of small birds. Avian Sci 2001, 1:15-23.

22. Anderson DR, Burnham KP: AIC model selection in overdispersed capture-recapture data. Ecology 1994, 75:1780-1793.

23. White GC, Burnham KP: Program MARK: survival estimation from populations of marked animals. Bird Study 1999, 46(Suppl):S120-S139.

doi:10.1186/2050-3385-2-2

Cite this article as: Naef-Daenzer and Grüebler: Effects of radio-tag characteristics and sample size on estimates of apparent survival. Animal Biotelemetry 2014 2:2.

\section{Submit your next manuscript to BioMed Central and take full advantage of:}

- Convenient online submission

- Thorough peer review

- No space constraints or color figure charges

- Immediate publication on acceptance

- Inclusion in PubMed, CAS, Scopus and Google Scholar

- Research which is freely available for redistribution 